\title{
The WISE Census of Young Stellar Objects and Clusters in Canis Major
}

\author{
W. J. Fischer ${ }^{1,2}$, D. L. Padgett ${ }^{1}$, and K. R. Stapelfeldt ${ }^{1}$ \\ ${ }^{1}$ NASA Goddard Space Flight Center \\ email: william.j.fischer@nasa.gov \\ ${ }^{2}$ NASA Postdoctoral Program Fellow
}

\begin{abstract}
The photometric data returned by WISE, the Wide-field Infrared Survey Explorer, can be used to search the sky for young stellar objects (YSOs) away from the molecular clouds studied in detail by Spitzer and Herschel. We present updated results for a $100 \mathrm{deg}^{2}$ region centered on Canis Major, including a look at the clustering properties of YSOs in the region.
\end{abstract}

Keywords. protoplanetary disks - stars: formation - stars: protostars

\section{Introduction}

Spitzer and Herschel teams found thousands of YSOs in the nearest kiloparsec, but searches were focused on the densest parts of molecular cloud complexes. WISE (Wright et al. 2010) surveyed the entire sky at $3.4,4.6,12$, and $22 \mu \mathrm{m}$, allowing a search for examples of low-mass star formation elsewhere. This will allow a better characterization of star and planet formation in regions with low initial gas densities. Here we present a study of a $100 \mathrm{deg}^{2}$ field centered on Canis Major. Large-scale studies of moderately distant star-forming regions help to bridge the gap from nearby clouds to external galaxies.

Star formation in Canis Major is centered just below the Galactic plane near $\ell=224^{\circ}$ and $b=-2^{\circ}$ at a distance of approximately $1000 \mathrm{pc}$ (Gregorio-Hetem 2008). Kim et al. (2004) mapped ${ }^{13} \mathrm{CO}$ gas in the region and found 22 clouds of median density $21 \mathrm{M}_{\odot} \mathrm{pc}^{-2}$, much lower than the median of $85 M_{\odot} \mathrm{pc}^{-2}$ in Orion (Nagahama et al. 1998). Parts of the region were recently mapped by Spitzer and Herschel surveys of the outer galactic plane.

We searched WISE for Class I YSOs (protostars with envelopes) and Class II YSOs (more evolved objects with with dusty disks but no envelopes). Due to slightly adjusted search criteria, counts presented here supersede those of Fischer et al. (2015).

\section{YSO Selection Techniques}

We centered our search at $106.67^{\circ} \mathrm{RA}$ and $-11.29^{\circ} \mathrm{Dec}$, the approximate center of the CMa OB1 association. We required WISE detections in $W 1$ and $W 2$, and we ignored sources flagged as artifacts in WISE or as extended sources in 2MASS. We adopted the WISE color-color criteria for YSOs of Koenig \& Lesiawitz (2014). These are calibrated with the colors of known YSOs in Taurus and galactic and extragalactic contaminants.

To eliminate a faint, uniformly distributed population of objects that are likely extragalactic, we required $W 1<12 \mathrm{mag}$ or $W 4<5$ mag. Bright galactic contaminants such as red giants were eliminated by requiring $W 1>6$ mag. Of the sources with the requisite colors, 146 Class I and 343 Class II candidates satisfy the magnitude requirements. These are shown in the left panel of Figure 1.

\section{Clusters of YSOs}

We connected the candidates with their minimum spanning tree (Gutermuth et al.2009) and retained links shorter than a critical length of $330^{\prime \prime}(1.6 \mathrm{pc})$. This leaves 56 clusters of sources; we plot those with more than four members in the right panel of Figure 1. The clusters are colored by their ratios of Class II to Class I sources, which range from 0.4 to 8.7 and serve 

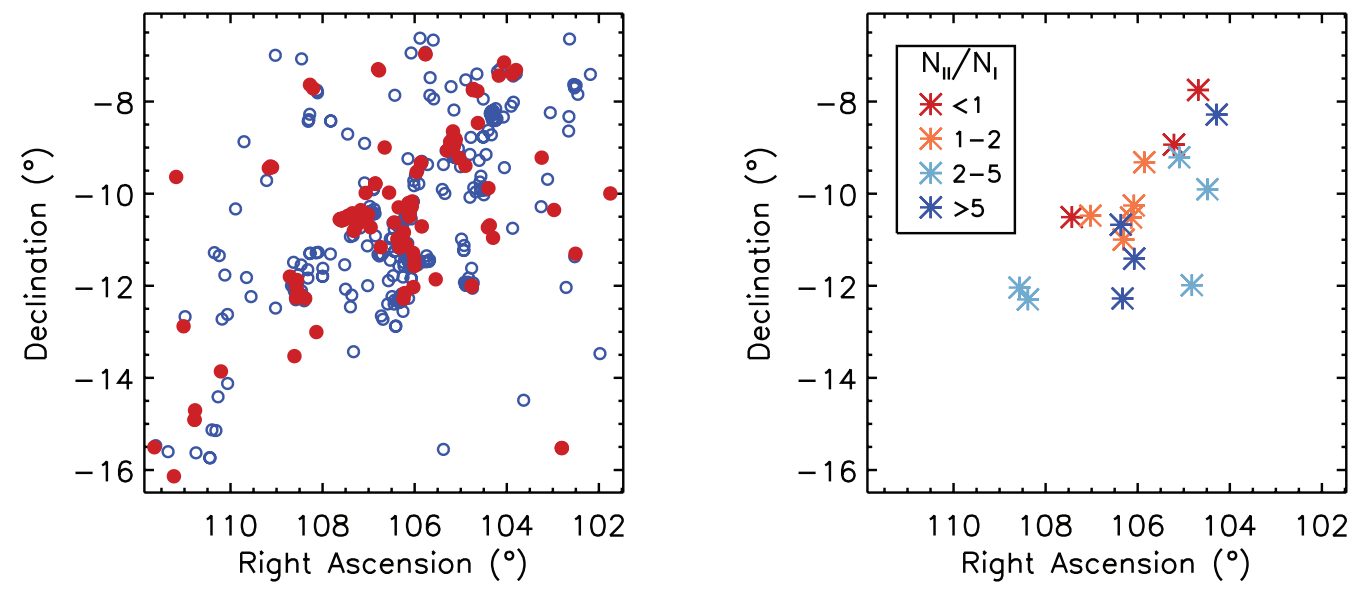

Figure 1. Left: YSO candidates in the $100 \mathrm{deg}^{2}$ surrounding Canis Major. Red filled circles are Class I candidates, while blue filled circles are Class II candidates. Right: Central locations of YSO clusters exceeding four members. The coloring indicates the Class II to Class I ratio, a proxy for age, which roughly increases from the northeast to the southwest. See the electronic edition for full color.

as a proxy for age (Myers 2012). The ratios and presumably the ages are smallest along the northeastern edge of the distribution, and older clusters are found to the west and south. The largest cluster, at $107.4^{\circ} \mathrm{RA}$ and $-10.5^{\circ}$ Dec, contains 42 members, 29 of which are Class I. It is an example of recent star formation far from famous molecular clouds.

\section{Conclusions}

With WISE photometric criteria, we detected 489 candidate YSOs in a $10^{\circ} \times 10^{\circ}$ square centered on the CMa OB1 association. Our clustering criteria select 17 clusters of more than four members; these appear to increase in age from northeast to southwest. We have acquired time in 2015 December and 2016 January for spectroscopy of the sources to verify their nature as YSOs and determine their spectral types and accretion properties.

\section{References}

Fischer, W.J., Padgett, D.L., \& Stapelfeldt, K. L. 2015, in Proceedings of IAU Symposium No. 314, J.H. Kastner, B. Stelzer, \& S.A. Metchev, eds.

Gregorio-Hetem, J. 2008, in Handbook of Star Forming Regions, B. Reipurth, ed., p. 1

Gutermuth, R.A., Megeath, S.T., Myers, P.C., et al. 2009, ApJS, 184, 18

Kim, B.G., Kawamura, A., Yonekura, Y., \& Fukui, Y. 2004, PASJ, 56, 313

Koenig, X.P., \& Leisawitz, D. T. 2014, ApJ, 791, 131

Myers, P. C. 2012, ApJ, 752, 9

Nagahama, T., Mizuno, A., Ogawa, H., \& Fukui, Y. 1998, AJ, 116, 336

Wright, E.L., Eisenhardt, P.R.M., Mainzer, A.K., et al. 2010, AJ, 140, 1868 